\title{
Identifying Early Predictive Markers for Immune-Related Adverse Events in Nivolumab-Treated Patients with Renal Cell Carcinoma and Gastric Cancer
}

\author{
Shinya Takada ${ }^{1 *}$, Hidetaka Murooka ${ }^{1}$, Kanae Tahatsu ${ }^{1}$, Maki Yanase ${ }^{1}$, Kengo \\ Umehara $^{1}$, Hirokazu Hashishita ${ }^{1}$, Harabayashi Toru ${ }^{2}$, Maruyama Satoru ${ }^{2}$, \\ Tamotsu Sagawa $^{3}$, Koshi Fujikawa ${ }^{3}$, Hideki Sato ${ }^{4}$, Kozo Mino $^{1}$
}

\begin{abstract}
Background: Neutrophil-to-lymphocyte (NLR) and platelet-to-lymphocyte (PLR) ratios can indicate poor disease prognosis and are inflammation markers. We investigated the role of NLR and PLR as effective predictive markers of immune-related adverse event (irAE) onset in patients treated with nivolumab. Methods: We retrospectively analysed 73 gastric and renal cancer patients treated with nivolumab at the Hokkaido Cancer Centre from January 2017 to June 2020. NLR and PLR were calculated at the initiation of nivolumab treatment and irAE onset. We identified the risk factors for Grade 3-4 irAE onset using NLR, PLR, sex, cancer type, and age. Overall survival (OS) and progression free survival (PFS) were calculated from the initiation of nivolumab treatment to the date of death or censored at last follow-up. Results: Among the 73 patients included, 17 (18\%) had at least one grade3-4 irAE. Multivariable logistic regression analyses revealed that pretreatment NLR $<4.3$ was significantly associated with a reduced risk for onset of grade3-4 irAEs, whereas rate of NLR change after treatment, $\triangle \mathrm{NLR}>120 \%$ was significantly associated with an increased risk. Conclusions: NLR is an effective marker for prognosis and onset of grade 3-4 irAEs.
\end{abstract}

Keywords: Immune checkpoint inhibitors- neutrophils to lymphocytes- platelet to lymphocytes

Asian Pac J Cancer Prev, 23 (2), 695-701

\section{Introduction}

Immune checkpoint inhibitors (ICIs) are currently being used in the treatment of a variety of cancers. Although ICIs are mostly used in the form of monotherapies, their use in combination with chemotherapy is also being evaluated. ICI can induce immune-related adverse events (irAEs) including pneumonitis, hepatitis, rash, colitis, and endocrinopathies (Boutros et al., 2016; Friedman et al., 2016). The systemic immune hyperreactivity caused by ICI leads to irAEs and in various diseases treated with ICI, the development of irAEs is associated with improved survival (Haratani et al., 2018; Masuda et al., 2019).

Studies conducted on the use of neutrophil-tolymphocyte (NLR) and platelet-to-lymphocyte (PLR) ratios confirmed their applicability as indexes of therapeutic effects in various cancers (Kiriu et al., 2018; Li et al., 2019; Khunger et al., 2018; Scilla et al., 2017; Katayama et al., 2019). For better application of the ICIs, it is essential to identify predictors of ICI effects and the onset of irAEs. The tumour microenvironment consists of inflammatory cells that play essential roles in tumorigenesis and tumour growth and are important factors in non-small cell lung cancer (Rivas-Fuentes et al., 2015). Therefore, inflammation plays an important role in tumour growth and development (Greten et al., 2019; Luborsky et al., 2020; Cursano et al., 2020). Recently, as inflammation markers, NLR and PLR were used to identify tumour-related inflammation (Kiriu et al., 2018; Li et al., 2019; Khunger et al., 2018; Scilla et al., 2017; Katayama et al., 2019). Moreover, they were proposed as simple indicators of the systemic inflammatory response in critically ill patients of breast, colon, and lung cancer (Zahorec et al., 2001). A previous study also reported that the baseline NLR value (i.e., NLR $\leq 5$ ) is associated with improved survival in lung cancer patients treated with nivolumab (Bagley et al., 2017). Although the baseline NLR value is useful for stratifying patients before nivolumab treatment, predictive biomarkers that enable clinicians to determine whether 
nivolumab treatment should be continued are unknown. In a study based on patients with lung cancer treated with nivolumab, the effect-accuracy were compared before and after treatment using an NLR cut-off value of 5 (Pavan et al., 2019). Furthermore, one report compared not only the overall survival (OS) with the cut-off value of NLR before treatment as 5 but also compared OS with NLR after 2 courses of treatment with the same cut-off value (Kiriu et al., 2018). Based on the above findings and the effect of ICI treatment on patient immunological status, the time-dependent change in NLR is hypothesized as a marker for predicting therapeutic ICI effects. However, the relationship between the intensity of inflammation up to irAEs onset, and the time-dependent change in NLR has not been clarified. In this study, we hypothesised that the NLR and PLR change during irAEs onset. To investigate this hypothesis, we focused on the time-dependent changes in NLR and PLR during treatment, rather than focussing on NLR and PLR at specific stages of treatment. This study specifically aimed to determine whether time-dependent changes in NLR and PLR are important for determining the onset of irAEs in patients with gastric cancer and renal cell cancer treated with nivolumab.

\section{Materials and Methods}

\section{Patients}

Consecutive patients who received nivolumab treatment at Hokkaido Cancer Centre (Japan) between January 2017 and June 2020 were enrolled retrospectively. A minimum follow-up duration of 3 months was required for patient inclusion in the study because of the higher probability of developing irAEs within the first 12 weeks of treatment (Haanen et al., 2017). Nivolumab therapy was administered until the occurrence of the following: disease progression, unacceptable toxicity, withdrawal, or death. The observation period of irAEs began at the start of nivolumab treatment to one month after nivolumab discontinuation. The toxicity was evaluated based on the Common Toxicity Criteria for Adverse Events, version 4.0. IrAEs were defined as pneumonitis, skin-related events (e.g., rash), diarrhoea, arthralgias/myalgias, endocrinerelated events (e.g., hypothyroidism, hypophysis, adrenal crisis), hepatitis, tubulointerstitial nephritis, and other conditions as previously described (Weber et al., 2012). The following inclusion criteria were used: the availability of clinical data and treatment with nivolumab based on clinical practice. The exclusion criteria included patients for whom the details of irAEs could not be confirmed or for whom clinical laboratory data were missing. This study was conducted in accordance with the Declaration of Helsinki and requirements of the institutional review board and was approved by the ethics committee of Hokkaido Cancer Centre, Sapporo, Japan (approval No. R2-25). All patients provided written informed consent to participate in the study.

\section{Study endpoint}

The primary endpoint for the study was the relationship between the onset of grade 3-4 irAE and the peripheral blood cell count. The peripheral blood cell counts were evaluated before the start of nivolumab treatment to determine the baseline value and upon irAE onset, from which the rate of change was calculated. When irAEs were absent, the value at the time of treatment discontinuation or 6 months after the start of treatment was used. The usefulness of the peripheral blood cell count as a systemic inflammatory biomarker has been reported in several studies (Kiriu et al., 2018; Li et al., 2019; Khunger et al., 2018; Scilla et al., 2017; Katayama et al., 2019), in which and NLR and PLR were calculated using the peripheral blood cell count. In this study, NLR and PLR before the start of treatment are presented as NLRpre and PLRpre, while the rates of change after treatment are expressed as $\triangle$ NLR and $\triangle$ PLR.

\section{Relationship between irAE onset and NLR, PLR, and survival time}

PFS and OS were calculated based on irAE onset of each grade. The irAE grades were divided into three groups (grade 0, grade 1-2 and grade 3-4), and the survival times were calculated for each group. In addition, as peripheral blood biomarkers, the cut-off values of NLRpre and PLRpre were calculated by performing receiver operating characteristic curve analysis and divided into two groups so that PFS and OS could be calculated, and the survival time compared.

\section{Statistical analysis}

For continuous variables, receiver operating characteristic (ROC) curve analysis was used to estimate the optimal cutoff values of adjusted age, NLR, PLR, $\triangle N L R$, and $\triangle P L R$ levels at baseline for the occurrence of grade 3 or higher irAEs.As another variable,the risk factors included gender, and cancer type (gastric cancer, renal cell carcinoma).Variables with potential association with the incidence of grade 3 or higher irAE suggested by univariate logistic regression analysis $(p<0.20)$ were considered when constructing the multivariate model. Results with two-sided $\mathrm{p}<0.05$ were considered statistically significant. Next, for survival (OS and PFS), three comparisons were made. The first comparison was NLRpre $<4.3$ and NLRpre $\geq 4.3$. The second comparison included PLRpre $<210$ and PLRpre $\geq 210$. The third comparison was overall survival according to the worst grade of irAE in ICI treatment. Log-rank analysis was Log-rank analysis was performed using the Kaplan-Meier method. Log-rank analysis was performed using the Kaplan-Meier method. All analyses were performed using BellCurve for Excel (Social Survey Research Information Center, Tokyo, Japan).

\section{Results}

\section{Patient background}

A total of 73 patients were included in this study, and the characteristics of renal and gastric cancers are summarized in Table 1. The median age of the patients was 67 years (IQR: 60-73); the cohorts included 54 males (74\%), 41 patients with renal cancer $(56 \%)$, and 32 patients with gastric cancers (44\%). The number of patients who received first, second-, and third-line treatments was 

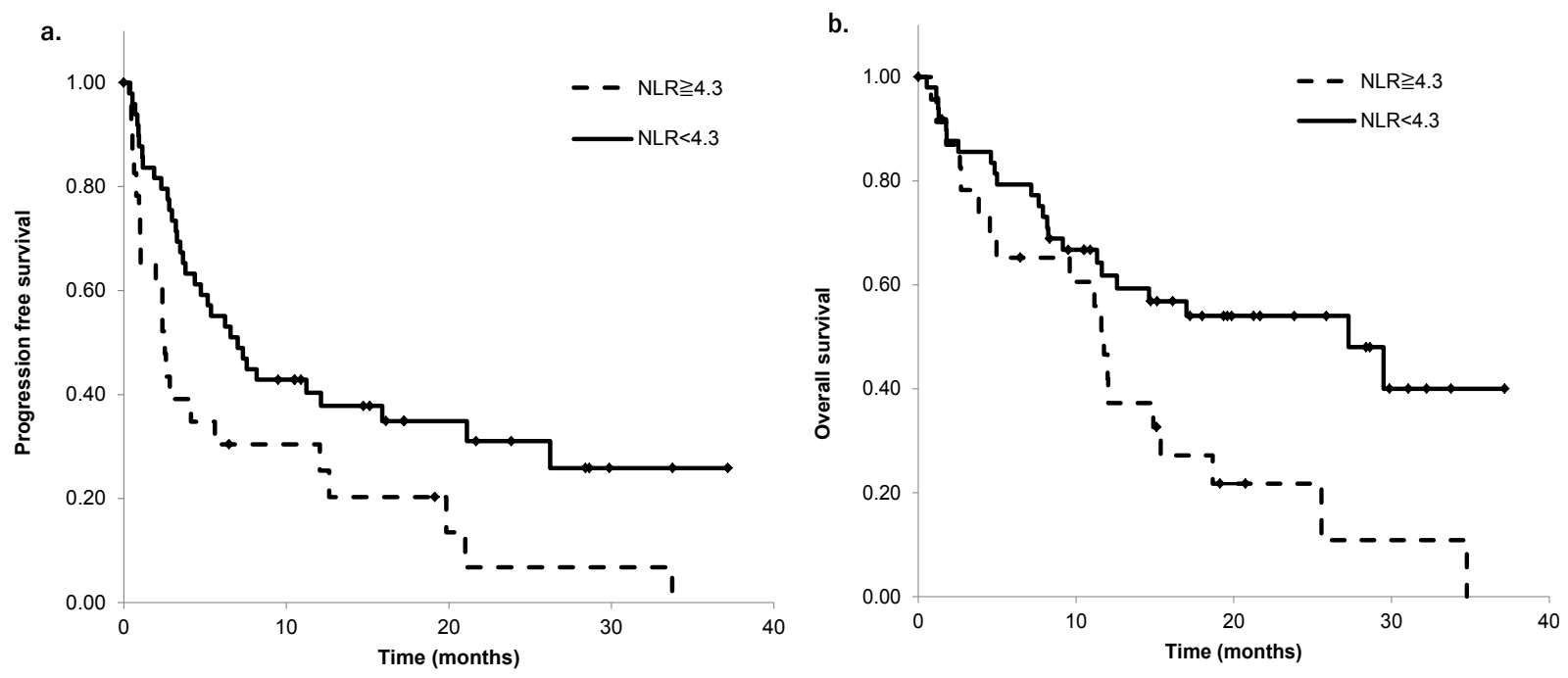

Figure 1. Kaplan-Meier Survival Analysis Based on NLR. Patients were stratified into two groups: patients with NLR $<4.3$ and NLR $\geq 4.3$. Baseline (NLRpre $<4.3$ ) groups are indicated with solid lines and NLR $\geq 4.3$ groups are indicated with dashed lines. Patients with low NLR pre had good prognosis; (a) shows PFS and (b) shows OS. NLR, Neutrophil-to-lymphocyte ratio; PFS, progression free survival; OS, overall survival;

Table 1. Baseline Characteristics of Patients

\begin{tabular}{ccc}
\hline & Gastric cancer & Renal cell carcinoma \\
\hline Age (years) & & \\
Median & 64 & 69 \\
Range & $41-88$ & $40-79$ \\
Gender $;$ Number(\%) & $30(73 \%)$ \\
Male & $24(75 \%)$ & $11(27 \%)$ \\
Female & $8(25 \%)$ & \\
Number of prior treatment line & 3 \\
$\leq 2$ & 26 & 29 \\
$>3$ & 15 &
\end{tabular}

$10(14 \%), 19(26 \%)$, and $44(60 \%)$, respectively. The cut-off values for NLRpre and PLRpre were 4.3 and 210, while those for $\triangle \mathrm{NLR}$ and $\triangle \mathrm{PLR}$ were $185 \%$ and $120 \%$, respectively.

\section{Immune-related adverse events}

Fifty-one patients $(70.0 \%)$ experienced seven different irAE types (Table 2). The median number of ICI administration was three (range, 1-42), and the median number of weeks before the onset of any
Table 2. Immune-Related Adverse Events among Cases of Gastric and Renal Cell Cancer

\begin{tabular}{lccc}
\hline irAE subtype & \multicolumn{2}{c}{ Grading, Number } & $\begin{array}{c}\text { Requiring } \\
\text { systemic } \\
\text { steroid } \\
\text { treatment, } \mathrm{n}(\%)\end{array}$ \\
\hline Skin-related events & 15 & 3 & $9(50 \%)$ \\
Pneumonitis & 3 & 5 & $6(75 \%)$ \\
Endocrine-related events & 1 & 0 & $1(100 \%)$ \\
Increased transaminase & 9 & 5 & $6(43 \%)$ \\
Fever & 2 & 0 & $0(0 \%)$ \\
Diarrhea & 3 & 2 & $3(60 \%)$ \\
Thyroid dysfunction & 12 & 1 & $0(0 \%)$ \\
other & 3 & 1 & $0(0 \%)$ \\
\hline
\end{tabular}

irAEs was 4.1 (range, $0.4-88.9$ weeks). Thirty-seven patients $(50 \%)$ developed irAEs within 12 weeks of the first nivolumab administration. IrAEs observed in these patients were mainly mild (grade $1-2$ in $73 \%$ of cases). Twenty-six patients permanently discontinued nivolumab treatment due to toxicity or progressive disease. During the study, 23 (32\%) of patients experienced IrAEs such as pneumonitis $(n=6)$, diarrhoea $(n=3)$, increased transaminase $(n=6)$, or skin-related events $(n=7)$ that

Table 3.Univariate and Multivariate Analyses of the Risk Factors for the Incidence of Immune-Related Adverse Events

\begin{tabular}{|c|c|c|c|c|}
\hline \multirow[b]{2}{*}{ Characteristic } & \multicolumn{2}{|c|}{ Univariate analysis } & \multicolumn{2}{|c|}{ Multivariate analysis } \\
\hline & Odds Ratio( $95 \% \mathrm{CI})$ & P-value & Odds Ratio $(95 \% \mathrm{CI})$ & P-value \\
\hline Sex (male; 1 ,female; 0$)$ & $0.49(0.14-1.73)$ & 0.3 & & \\
\hline Cancer Type (renal cell carcinoma; 1 ,gastric cancer;0) & $0.30(0.083-1.08)$ & 0.07 & $5.00(0.72-34.70)$ & 0.1 \\
\hline Age $>70$ years $(>70 y ; 1, \leq 70 y ; 0)$ & $0.53(0.16-1.78)$ & 0.3 & & \\
\hline Neutrophil-to-lymphocyte ratio at baseline (NLR $<4.3 ; 1, \geq 4.3 ; 0)$ & $0.14(0.038-0.53)$ & 0.003 & $0.024(0.0012-0.46)$ & $0.014 *$ \\
\hline Platelet-to-lymphocyte ratio at baseline (PLR $<210 ; 1, \geq 210 ; 0)$ & $0.10(0.013-0.86)$ & 0.02 & $0.31(0.0028-3.41)$ & 0.34 \\
\hline Rate of change NLR after treatment $(\Delta \mathrm{NLR}>120 \% ; 1, \leq 120 \% ; 0)$ & $6.13(1.24-30.30)$ & 0.023 & $10.48(1.20-91.84)$ & $0.033^{*}$ \\
\hline Rate of change PLR after treatment $(\triangle \mathrm{PLR}>185 \% ; 1, \leq 185 \% ; 0)$ & $3.07(0.84-11.26)$ & 0.12 & $12.78(0.70-232.72)$ & 0.085 \\
\hline
\end{tabular}

CI, Confidence interval; *, Statistically significant 

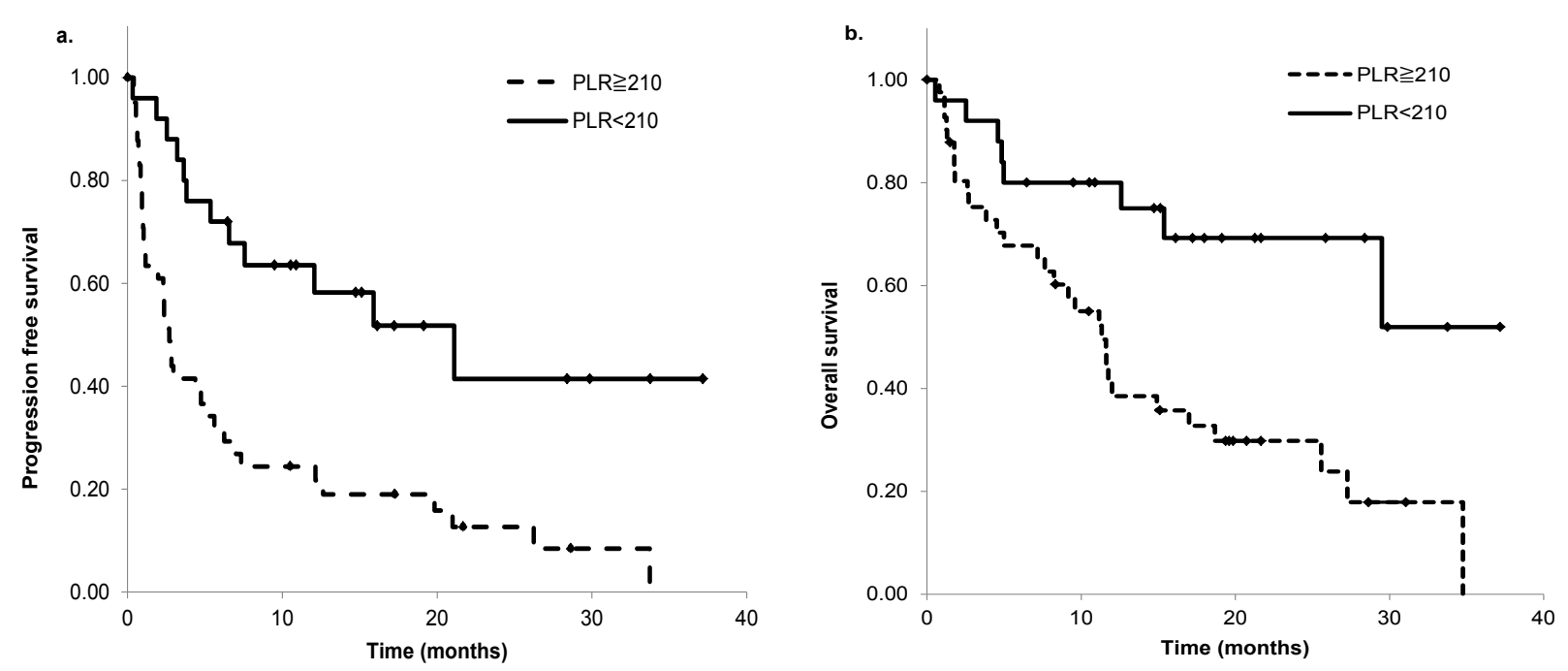

Figure 2. Kaplan-Meier Survival Analysis Based on PLR. Patients were stratified into two groups: patients with PLR $<210$ and patients with PLR $\geq 210$. Baseline (PLRpre $<210$ ) groups are indicated with solid lines and PLR $\geq 210$ groups are indicated with dashed lines. Patients with low PLRpre had good prognosis; (a) shows PFS and (b) shows OS. PLR, platelet-to-lymphocyte ratio; PFS, progression free survival; OS, overall survival

required immunosuppressants such as systemic or topical steroidal treatment. Additionally, one patient with an endocrine irAE required hormonal replacement therapy. Moreover, 18 patients started thyrostatic treatment for hyperthyroidism. These results indicated that frequency and type of irAEs were similar to those of previously reported irAEs.

\section{Relationship between NLRpre and PFS and OS}

Patients with lower NLRpre showed improved PFS and OS. Patients were categorized as those with NLRpre $\leq 4.3$ or $\geq 4.3$. Patients with a NLRpre $<4.3$ had a median PFS of 6.9 months (95\% CI 3.9-9.9) compared to that of 2.3 months (95\% CI 1.6-3.1) in patients with NLRpre $\geq 4.3(\mathrm{P}<0.01$, Figure 1a). Similarly, patients with NLRpre $<4.3$ had a median OS of 26.9 months $(95 \%$
CI 9.3-44.5) compared to that of 11.4 months (95\% CI 8.2-14.7) in patients with NLRpre $\geq 4.3(\mathrm{P}<0.01$, Figure 1b). These results showed that the low NLRpre group had a longer survival than the high NLRpre group.

\section{Relationship between PLRpre and PFS and OS}

Patients with lower PLRpre showed improved PFS and OS. Patients were divided into those with PLRpre $\leq 210$ or $\geq 210$. Patients with PLRpre $<210$ had a median PFS of 20.8 months (95\% CI 7.6-34.1) compared to that of 2.7 months (95\% CI 2.1-3.2) in patients with PLRpre $\geq 210$ $(\mathrm{P}<0.001$, Figure 2a). Similarly, patients with PLRpre $<210$ had a median OS of not reached. Patients with PLRpre $\geq 210$ had a median OS of 11.2 months $(95 \%$ CI 8.4-14.0) $(\mathrm{P}<0.01$, Figure 2b). These results showed that the low PLRpre group had longer survival than the
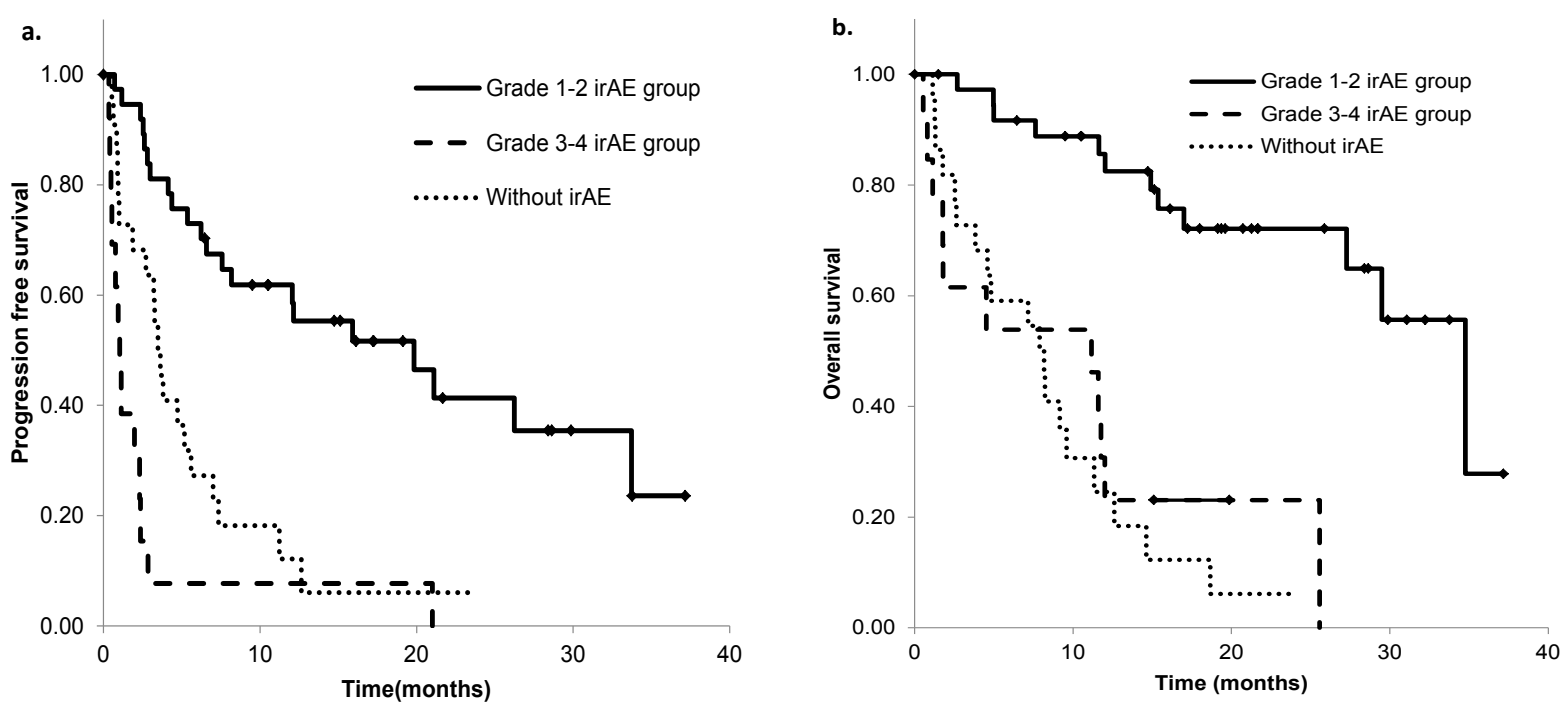

Figure 3. Overall Survival and Progression-Free Survival of the Patient Population Enrolled in this Study. (a)PFS and (b) OS according to the onset of immune related adverse events (irAEs). Solid line, grade 1-2 irAEs; dashed line, grade 3-4 irAEs; and dotted line, patients without irAEs. PFS, progression free survival; OS, overall survival 
high PLRpre group.

\section{Relationship between irAE grade and PFS and OS}

Based on a three-group comparison, the irAEs were divided into grade 0 , grade 1-2, and grade 3-4. Our analysis showed that the grade 1-2 group had significantly improved OS and PFS compared with the other two groups. The median OS for the grade 1-2 irAE group was 34.3 months $(95 \%$ CI $26.8-41.8)$, while it was 11.0 months for grade $3-4(95 \%$ CI $4.9-22.1)$ and 7.8 months for grade $0(95 \%$ CI 2.7 - 12.9; grade $1-2$ vs. grade $3-4$ and grade $0, p<0.001$ and $p<0.001$, respectively; Figure 3a). Similarly, the median PFS for the grade 1-2 irAE group was 19.6 months (95\% CI $7.8-31.3), 1.0$ months for grade $3-4(95 \%$ CI $0.6-1.4)$ and 3.4 months for grade $0(95 \%$ CI $2.8-4.1$; grade $1-2$ vs. grade $3-4$ and grade $0, p<0.001$ and $p<0.001$, respectively; Figure $3 b)$.These results showed that the grade1-2 group had a longest survival compared with the grade 0 and grade $3-4$ irAE groups.

\section{Multivariate analysis of factors associated with irAE incidence}

The results of the univariate and multivariate analyses are shown in Table 2. The cutoff values of continuous variables for univariate analysis were calculated by ROC analysis.

ROC curve analysis revealed that the cutoff of baseline NLR level was 4.3, NLR level was 120, PLR level was 210 , and PLR level was 185 with an area under the ROC curve of 0.70 (95\% confidence interval (CI); 0.52-0.88), 0.68 (95\% confidence interval (CI); $0.53-0.83), 0.62$ (95\% confidence interval (CI); 0.46-0.79), and $0.63(95 \%$ confidence interval (CI);0.49-0.77). Factors with a $\mathrm{P}$-value of 0.2 or less in the results of univariate analysis were used as factors for multivariate analysis. The factors identified in the univariate analysis were cancer type (RCC; $\mathrm{P}=0.07), \mathrm{NLR}(<4.3 ; \mathrm{P}=0.003), \mathrm{PLR}(<210$; $\mathrm{P}=0.02), \Delta \mathrm{NLR}(>120 \% ; \mathrm{P}=0.023)$, and $\triangle \mathrm{PLR}(>185 \%$; $\mathrm{P}=0.12)$. Multivariate analysis revealed that pretreatment NLR values (odds ratio, 95\% confidence interval: $0.003,0.001-0.46, p=0.014)$ and change after treatment initiation $(\triangle \mathrm{NLR})$ values (odds ratio, 95\% confidence interval: $10.48,1.20-91.84, \mathrm{p}=0.033$ ) are risk factors for grade 3-4 irAE onset.

\section{Discussion}

Our results collectively provide convincing evidence for the use of NLR and PLR as prognostic and predictive markers for irAE onset in response to nivolumab treatment in cancer patients. To the best of our knowledge, this is the first report to associate time-dependent changes in NLR and PLR with irAE onset in patients administered nivolumab treatment. Effective management of grade 3-4 irAEs associated with nivolumab treatment is especially important. Treatment of grade 3-4 irAEs usually requires hospitalisation because they can result in decreased quality of life (QOL) or cause fatality. Although associated risk factors such as a history of autoimmune disease are known, biochemical markers that can predict the risk of irAEs associated with nivolumab treatment have not yet been identified (Hopkins et al., 2017; Champiat et al., 2016). Nivolumab can modulate patient immunity by altering various inflammatory markers, such as NLR, PLR, and monocyte-to-lymphocyte ratio (Liu et al.,2019; Yodying et al., 2016; Mirili et al., 2019). Among these markers, NLR and PLR have been reported as effective markers for survival. NLR and PLR are markers of systemic inflammation and are expected to fluctuate since enhanced inflammation is observed following nivolumab treatment (Liu et al., 2019; Yi et al., 2018). In fact, in autoimmune diseases such as rheumatoid arthritis and Crohn's disease, NLR and PLR were reportedly significantly higher than in autoimmune disease-free groups (Liu et al., 2020; Feng et al., 2017; Gao et al., 2015; Erre et al., 2019). For example, the NLR in Crohn's disease (5.72 \pm 6.66$)$ was significantly higher compared to that of the control group (1.84 \pm 0.85$)$ (Gao et al., 2017). Therefore, we hypothesised that NLR and PLR would increase in response to nivolumab-triggered irAEs in a hyperreactive immunological state. We also aimed to identify changes in NLR and PLR that could be used to predict irAE, as well as the time-dependent changes in NLR and PLR that occur before irAE onset. Baseline NLR and PLR are known markers for improved OS; therefore, various cut-off values have been set for NLR, among which 3 to 5 are commonly reported (Nakaya et al., 2018; Ackland et al., 2019; Iwase et al., 2017; Kiriu et al., 2018). These reports have also suggested that OS and PFS are significantly prolonged in patients with low NLR before the start of treatment (Kiriu et al., 2018; Li et al., 2019; Khunger et al., 2018; Scilla et al., 2017; Katayama et al., 2019). In our study, the cut-off of each NLR and PLR value before the start of treatment was set to 4.3 and 210 , respectively, and OS and PFS were compared between the low group (NLRpre and PLRpre; <4.3 and <210) and high groups (NLRpre and PLRpre; $\geq 4.3$ and $\geq 210$ ). Our results showed that the low-NLR and -PLR groups showed better OS and PFS. This finding was consistent with that reported earlier in stage 4 non-small cell lung and renal cancer (Bagley et al., 2017; Kazuo et al., 2020). Our study focused on the time-dependent changes in NLR and PLR from the initiation of nivolumab treatment to the onset of irAEs and identified markers that could predict irAE occurrence. The complete blood count (CBC) data were used to calculate NLR (absolute neutrophil count/absolute lymphocyte count) and PLR (platelet count/lymphocyte count). The advantage of using NLR and PLR is that they can be used for long-term follow-up. Collectively, our results suggested that the time-dependent changes in NLR can serve as a useful marker for predicting the development of serious irAEs in response to immunotherapy. As an inflammatory marker, an elevated NLR may be associated with severe irAE onset and disease progression. In the elevated NLR group ( $\geq 120 \%$ ), 84\% of patients had to discontinue nivolumab due to progressive disease (PD-or irAE. Therefore, our results confirmed that NLR is affected by nivolumab treatment and showed that if NLR increased by $120 \%$ or more, it could be an indicator of poor prognosis.

It has been reported that irAEs onset by ICI improves

Asian Pacific Journal of Cancer Prevention, Vol 23 
OS and PFS. However, no comparison of irAE severity has been reported to the best of our knowledge. Therefore, the irAE onset group was divided into grade 1-2 and grade 3-4 groups to evaluate how irAE severity was related to OS and PFS. We found that the patients with grade 1-2 irAEs had significantly improved OS and PFS compared to the other two groups (Figure 3). However, the OS and PFS of the patients with irAEs of grade 3-4 did not improve compared to the grade 0 despite irAE onset. Onset of grade 3-4 irAEs occurred early during the start of treatment and interrupted the regimen so that a sufficient therapeutic effect could not be obtained. In fact, in patients with grade 3-4 irAEs, the median duration of irAE onset was 37 days after treatment, at which point, irAE onset accounted for treatment discontinuation in $100 \%(13 / 13)$ of the cases. Regarding the incidence of grade 3-4 irAEs, NLRpre < 5 and an NLRpre increase of more than $120 \%(\triangle N L R$ $>120 \%$ ) were identified as significant risk factors of grade3-4 irAE onset using multivariate logistic regression analysis (Table 3). Mingjia et al. observed that the increased NLR was also a significantly poor predictor of survival (Li et al., 2019). Thus, overall, an increased NLR predicts poor outcomes for patients. Moreover, Pavan et al. reported that the NLR and PLR were also significant predictors of irAE development (Pavan et al., 2019). Development of ICI-induced irAEs possibly reflects the hyperactivity of $\mathrm{T}$ cells, owing to them being unblocked and reactivated by ICIs. Future studies should validate this hypothesis by identifying a link between cytokine and chemokine expression and time-dependent changes in NLR.

Our study had some limitations. First, it was a retrospective pilot study to clarify the relationship between irAEs onset and NLR/change of $\operatorname{NLR}(\triangle N L R)$ and PLR/ change of PLR( $\triangle \mathrm{PLR})$. Second, we limited our study to grade 3-4 irAEs that required steroid therapy. Thus, our study outcomes propose that NLRpre and $\triangle$ NLR may be the predictive markers for onset of Grade 1-2 irAEs. However, this time, we focused on Grade 3-4 irAEs, which affect the continuation of treatment. In future, it will be necessary to perform large-scale prospective studies targeting irAEs of all grades. Third, the confirmation of irAE onset was limited to the information obtained from the initiation to one month after nivolumab treatment. It cannot be ruled out that some of the late onset irAE related information may have been missed within this time period.

In conclusion, this is the first study to focus on the time-dependent changes in NLR and investigate the possibility of using NLR as a predictive marker for irAE onset. In the future, NLR may not only be used as an important marker of the onset of grade 3-4 irAEs but also a marker for predicting the efficacy of nivolumab treatment. Future studies will explore how these markers can be utilised for evaluating the efficacy and outcomes of nivolumab treatment.

\section{Author Contribution Statement}

All authors discussed the results and commented on the manuscript. TS, HH, TH, SM, S T, and FK confirmed medical judgment and designed this study. ST, MH, TK,
YM, and UK provided advice on statistical analysis. ST, $\mathrm{SH}$ and MK edited the manuscript.

\section{Acknowledgements}

We would like to express our gratitude to all the researchers of Hokkaido Cancer Center who participated in this study.

Ethics approval and consent to participate

This study was approved by the ethics committee of Hokkaido Cancer Center (Approval No: R2-25). All patients provided written informed consent to participate in the study.

\section{Data availability}

The data supporting the findings of this study are available from the corresponding author, [ST], upon reasonable request.

\section{Conflict of interest}

Authors declare no conflict of interest.

\section{References}

Ackland GL, Abbott, TEF, Cain D, et al (2019). Preoperative systemic inflammation and perioperative myocardial injury: prospective observational multicentre cohort study of patients undergoing non-cardiac surgery. Br J Anaesth, 122, 180-7.

Bagley SJ, Kothari S, Aggarwal C, et al (2017). Pretreatment neutrophil-to-lymphocyte ratio as a marker of outcomes in nivolumab-treated patients with advanced non-small-cell lung cancer. Lung Cancer, 106, 1-7.

Boutros C, Tarhini A, Routier E, et al (2016). Safety profiles of anti-CTLA-4 and anti-PD-1 antibodies alone and in combination. Nat Rev Clin Oncol, 13, 473-86.

Champiat S, Lambotte O, Barreau E, et al (2016). Management of immune checkpoint blockade dysimmune toxicities: a collaborative position paper. Ann Oncol, 27, 559-74.

Cursano MC, Kopf B, Scarpi E, et al (2020). Prognostic role of systemic inflammatory indexes in germ cell tumors treated with high-dose chemotherapy. Front Oncol, 10, 1325

Erre GL, Paliogiannis P, Castagna F, et al (2019). Meta-analysis of neutrophil-to-lymphocyte and platelet-to-lymphocyte ratio in rheumatoid arthritis. Eur J Clin Invest, 49, e13037.

Feng JR, Qiu X, Wang F, et al (2017). Diagnostic value of neutrophil-to-lymphocyte ratio and platelet-to-lymphocyte ratio in Crohn's disease. Gastroenterol Res Pract, 2017, 3526460.

Friedman CF, Proverbs-Singh TA, Postow MA(2016). Treatment of the immune-related adverse effects of immune checkpoint inhibitors: a review. JAMA Oncol, 2, 1346-53.

Gao SQ, Huang LD, Dai RJ, et al (2015). Neutrophil-lymphocyte ratio: a controversial marker in predicting Crohn's disease severity. Int J Clin Exp Pathol, 8, 14779-85.

Greten FR, Grivennikov SI (2019). Inflammation and cancer: triggers, mechanisms, and consequences. Immunity, 51, $27-41$.

Haanen JBAG, Carbonnel F, Robert C, et al (2017). Management of toxicities from immunotherapy: ESMO clinical practice guidelines for diagnosis, treatment and follow-up. Ann Oncol, 28, iv119-42.

Haratani K, Hayashi H, Chiba Y, et al (2018). Association of immune-related adverse events with nivolumab efficacy in non-small-cell lung cancer. JAMA Oncol, 4, 374-8. 
Hopkins AM, Rowland A, Kichenadasse G, et al (2017). Predicting response and toxicity to immune checkpoint inhibitors using routinely available blood and clinical markers. Br J Cancer, 117, 913-20.

Iwase T, Sangai T, Sakakibara Mg et al (2017). An increased neutrophil-to-lymphocyte ratio predicts poorer survival following recurrence for patients with breast cancer. $\mathrm{Mol}$ Clin Oncol, 6, 266-70.

Katayama Y, Shimamoto T, Yamada T, et al (2019). Retrospective efficacy analysis of immune checkpoint inhibitor rechallenge in patients with non-small cell lung cancer. J Clin Med, 9, 102.

Kazuo K, Yusuke I, Makoto H, et al (2020). Association between immune-related adverse events and clinical outcome following Nivolumab treatment in patients with metastatic renal cell carcinoma. In Vivo, 34, 2647-52.

Khunger M, Patil PD, Khunger A, et al (2018). Post-treatment changes in hematological parameters predict response to nivolumab monotherapy in non-small cell lung cancer patients. PLoS One, 13, e0197743.

Kiriu T, Yamamoto M, Nagano T, et al (2018). The time-series behavior of neutrophil-to-lymphocyte ratio is useful as a predictive marker in non-small cell lung cancer. PLoS One, 13, e0193018.

Li M, Spakowicz D, Burkart J, et al (2019). Change in neutrophil to lymphocyte ratio during immunotherapy treatment is a non-linear predictor of patient outcomes in advanced cancers. J Cancer Res Clin Oncol, 145, 2541-6.

Liu J, Li S, Zhang S, et al (2019). Systemic immuneinflammation index, neutrophil-to-lymphocyte ratio, platelet-to-lymphocyte ratio can predict clinical outcomes in patients with metastatic non-small-cell lung cancer treated with nivolumab. J Clin Lab Anal, 33, e22964.

Liu P, Li P, Peng Z, et al (2020). Predictive value of the neutrophil-to-lymphocyte ratio, monocyte-to-lymphocyte ratio, platelet-to-neutrophil ratio, and neutrophil-tomonocyte ratio in lupus nephritis. Lupus, 29, 1031-9.

Luborsky J, Barua A, Edassery S, et al (2020). Inflammasome expression is higher in ovarian tumors than in normal ovary. PLoS One, 15, e0227081.

Masuda K, Shoji H, Nagashima K, et al (2019). Correlation between immune-related adverse events and prognosis in patients with gastric cancer treated with nivolumab. $B M C$ Cancer, 19, 974.

Mirili C, Y1lmaz A, Demirkan S, et al (2019). Clinical significance of prognostic nutritional index (PNI) in malignant melanoma. Int J Clin Oncol, 24, 1301-10.

Nakaya A, Kurata T, Yoshioka H, et al (2018). Neutrophil-to-lymphocyte ratio as an early marker of outcomes in patients with advanced non-small-cell lung cancer treated with nivolumab. Int J Clin Oncol, 23, 634-40.

Pavan A, Calvetti L, Dal Maso A, et al (2019). Peripheral blood markers identify risk of immune-related toxicity in advanced non-small cell lung cancer treated with immune-checkpoint inhibitors. Oncologist, 24, 1128-36.

Rivas-Fuentes S, Salgado-Aguayo A, Pertuz Belloso S, et al (2015). Role of chemokines in non-small cell lung cancer: Angiogenesis and inflammation. $J$ Cancer, 6, 938-52.

Scilla KA, Bentzen SM, Lam VK, et al (2017). Neutrophillymphocyte ratio is a rognostic marker in patients with locally advanced (stage IIIA and IIIB) non-small cell lung cancer treated with combined modality therapy. Oncologist, 22, 737-42.

Weber JS, Kähler KC, Hauschild A (2012). Management of immune-related adverse events and kinetics of response with ipilimumab. J Clin Oncol, 30, 2691-7.

Yi F, Gu Y, Chen S, et al (2018). Impact of the pretreatment or
Predictive Marker of Immune-Related Adverse Events

posttreatment NLR and PLR on the response of first line chemotherapy and the outcomes in patients with advanced non-small cell lung cancer. Zhongguo Fei Ai Za Zhi, 21, 481-92.

Yodying H, Matsuda A, Miyashita M, et al (2016). Prognostic significance of neutrophil-to-lymphocyte ratio and plateletto-lymphocyte ratio in oncologic outcomes of esophageal cancer: A systematic review and meta-analysis. Ann Surg Oncol, 23, 646-54.

Zahorec R (2001). Ratio of neutrophil to lymphocyte counts-rapid and simple parameter of systemic inflammation and stress in critically ill. Bratisl Lek Listy, 102, 5-14.

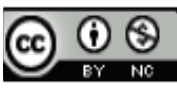

This work is licensed under a Creative Commons AttributionNon Commercial 4.0 International License. 